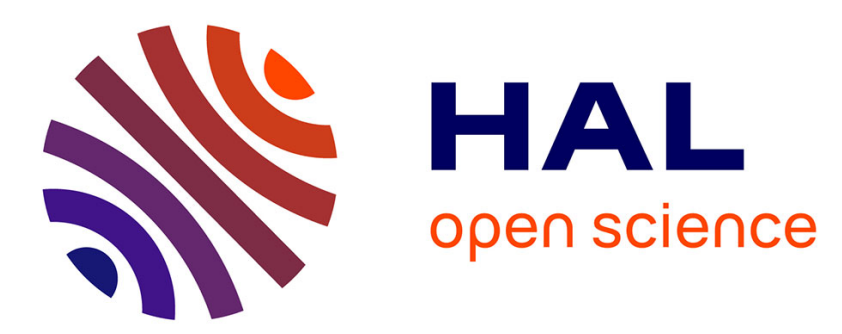

\title{
Temperature dependence of the radiative recombination coefficient in crystalline silicon by spectral and modulated photoluminescence
}

Rudolf Brüggemann, Ming Xu, José Alvarez, Mohamed Boutchich, Jean-Paul Kleider

\section{To cite this version:}

Rudolf Brüggemann, Ming Xu, José Alvarez, Mohamed Boutchich, Jean-Paul Kleider. Temperature dependence of the radiative recombination coefficient in crystalline silicon by spectral and modulated photoluminescence. physica status solidi (RRL) - Rapid Research Letters, 2017, 11 (6), 10.1002/pssr.201700066 . hal-01631792

\section{HAL Id: hal-01631792}

https://hal-centralesupelec.archives-ouvertes.fr/hal-01631792

Submitted on 20 Mar 2020

HAL is a multi-disciplinary open access archive for the deposit and dissemination of scientific research documents, whether they are published or not. The documents may come from teaching and research institutions in France or abroad, or from public or private research centers.
L'archive ouverte pluridisciplinaire HAL, est destinée au dépôt et à la diffusion de documents scientifiques de niveau recherche, publiés ou non, émanant des établissements d'enseignement et de recherche français ou étrangers, des laboratoires publics ou privés. 


\title{
Temperature dependence of the radiative recombination coefficient in crystalline silicon by spectral and modulated photoluminescence
}

\author{
Rudolf Brüggemann*, Ming Xu, José Alvarez, Mohamed Boutchich, and Jean-Paul Kleider \\ GeePs (Group of Electrical Engineering - Paris), UMR CNRS 8507, CentraleSupélec, Univ. Paris-Sud, Université Paris-Saclay, \\ Sorbonne Universités, UPMC Univ Paris 06, 11 rue Joliot-Curie, Plateau de Moulon, F-91192 Gif-sur-Yvette Cedex, France
}

Received 10 March 2017, revised 29 March 2017, accepted 7 April 2017

Published online 19 April 2017

Keywords photoluminescence, radiative recombination, recombination coefficient, silicon

*Corresponding author: e-mail rudi.brueggemann@geeps.centralesupelec.fr, Phone: +33 (0)169851645, Fax: +33 (0)169418318

By the combination of temperature-dependent spectral photoluminescence with modulated photoluminescence measurements the band-to-band radiative recombination coefficient is determined for crystalline silicon without the requirement to model the temperature dependence of the intrinsic carrier density, thus eliminating a source of error. By application of this approach we reproduce the variation of the radiative recombination coefficient in crystalline silicon with temperature reported in the literature for temperatures $\geq 77 \mathrm{~K}$. Above all, we extend the measured range for the radiative recombination coefficient to a temperature of $20 \mathrm{~K}$. In this extended temperature range between 20 and $70 \mathrm{~K}$ the recombination coefficient increases with decreasing temperature by about three orders of magnitude.
Radiative recombination is one of several recombination channels in semiconductors and its rate may be described by the product of the densities of the two recombining species and the radiative recombination coefficient $B_{\text {rad. }}$. The values of $B_{\text {rad }}$ are important in order to quantitatively describe the radiative recombination channel and also as input for device simulation, for example for modelling electroluminescence.

For crystalline silicon (c-Si), conflicting values from different evaluations were reviewed by Trupke et al. [1] who also determined $B_{\text {rad }}$ at several temperatures $T$ from 300 to $77 \mathrm{~K}$ [1]. Brüggemann et al. [2] confirmed those $T$-dependent $B_{\mathrm{rad}}-$ values and extended the measurement range to $393 \mathrm{~K}$ in which $B_{\text {rad }}$ is almost constant. Similar results were achieved by Nguyen et al. [3] who determined $B_{\text {rad }}$ in the $T$-range between 90 and $363 \mathrm{~K}$.

Previous microscopic calculations of $B_{\mathrm{rad}}$ of c-Si had been performed by Ruff et al. [4] to obtain the absolute values of the spectral distribution and of its $T$-dependence. The microscopic theory showed the trend in the $T$-dependence of $B_{\text {rad }}$, namely that it is almost constant in the range between about 400 and $250 \mathrm{~K}$ with increasing values with further decreasing $T$ and with a value of $7 \times 10^{-14} \mathrm{~cm}^{3} \mathrm{~s}^{-1}$ at the lowest $T$ of $90 \mathrm{~K}$ [4].

As reviewed and outlined by Trupke et al. [1] the energy ( $\hbar \omega)$ - and $T$-dependent spectral $B_{\mathrm{rad}, \mathrm{s}}(\hbar \omega, T)$ may be determined from the energy-dependent absorption coefficient for band-to-band transitions $\alpha_{B B}(\hbar \omega)$ and other quantities. Integrated appropriately over energy leads to the total recombination coefficient $B_{\mathrm{rad}}(T)$ as

$$
B_{\mathrm{rad}}(T)=\int B_{\mathrm{rad}, \mathrm{s}}(T, \hbar \omega) \mathrm{d}(\hbar \omega)
$$

One problem that arises is that $\alpha_{B B}$ is very small at low $T$ and $\hbar \omega$ [4]. Also, the square of the intrinsic carrier density $n_{i}$ enters the equation for $B_{\text {rad,s. }}$ The accuracy of these data thus critically influences the accuracy of $B_{\mathrm{rad}}$ at different $T$. It is also noted that despite long-time research on c-Si, no values of $B_{\text {rad }}$ for $T<77 \mathrm{~K}$ are available in the literature.

In this letter, we close this gap and we determine $B_{\text {rad }}(T)$ in $\mathrm{c}-\mathrm{Si}$ by a combination of stationary spectral photoluminescence (sPL) and modulated photoluminescence 
(MPL) under identical photoexcitation conditions and at the same excitation spot on a c-Si wafer. The elegance of the method results from the fact that neither values of $\alpha_{B B}(\hbar \omega, T)$ nor of $n_{i}(T)$ are needed.

The spectral sPL-photon flux $\mathrm{d} j_{\mathrm{PL}}$ emitted from a sample surface is given by $[5,6]$

$$
\mathrm{d} j_{\mathrm{PL}}(T, \hbar \omega)=\frac{A(T, \hbar \omega) \mathrm{d} r_{s p}(T, \hbar \omega)}{4 \alpha_{B B}(T, \hbar \omega) n_{r}^{2}(T, \hbar \omega)}
$$

where $n_{r}(T, \hbar \omega)$ is the refractive index of the semiconductor and $A(T, \hbar \omega)$ the absorption of the sample. The equation for the spontaneous band-to-band spectral emission rate $\mathrm{d} r_{s p}$ is expressed by

$$
\mathrm{d} r_{s p}(T, \hbar \omega)=B_{\text {rad,s }}(T, \hbar \omega) n p \mathrm{~d}(\hbar \omega),
$$

where $n$ and $p$ represent the recombining species via the free electron and hole densities in the bands. It links $B_{\text {rad,s }}(T, \hbar \omega)$ with the measured PL radiation. The spectral radiative recombination coefficient $B_{\text {rad,s }}(T, \hbar \omega)$ can thus be determined by

$$
B_{\mathrm{rad}, \mathrm{s}}(T, \hbar \omega) \mathrm{d}(\hbar \omega)=\frac{\mathrm{d} j_{\mathrm{PL}}(T, \hbar \omega)}{n p} \times \frac{4 \alpha_{B B}(T, \hbar \omega) n_{r}^{2}(T, \hbar \omega)}{A(T, \hbar \omega)} .
$$

For a wafer with front and rear surface reflection coefficient $R_{\text {front }}(\hbar \omega)$ and $R_{\text {rear }}(\hbar \omega), A$ takes account of reabsorption and multiple reflection of the PL radiation in the sample according to [7]

$$
\begin{aligned}
A(T, \hbar \omega) & =\left[1-R_{\text {front }}(\hbar \omega)\right]\left[1-\exp \left(-\alpha_{B B}(T, \hbar \omega) d\right)\right] \\
& \times \frac{\left[1+R_{\text {rear }} \exp \left(-\alpha_{B B}(T, \hbar \omega) d\right)\right]}{1-R_{\text {front }} R_{\text {rear }}(\hbar \omega) \exp \left(-2 \alpha_{B B}(T, \hbar \omega) d\right)},
\end{aligned}
$$

where $d$ is the sample thickness. Here, absorption other than by band-to-band transitions is neglected.

The equation for $A(T, \hbar \omega)$ and thus $B_{\text {rad }}(T, \hbar \omega)$ can be simplified for the condition that the product of $\alpha_{B B}(T, \hbar \omega) d$ is smaller than unity as is typically the case for c-Si at lower $T$. At around room temperature $\alpha_{B B}(T, \hbar \omega) d<1$ for most of the spectral range. Equation (4) then reads

$$
B_{\mathrm{rad}, \mathrm{s}}(T, \hbar \omega) \mathrm{d}(\hbar \omega)=\frac{\mathrm{d} j_{\mathrm{PL}}(T, \hbar \omega)}{n p} \frac{4 n_{r}^{2}(T, \hbar \omega)}{C_{\mathrm{ref}} d},
$$

where $C_{\text {ref }}$ subsumes reflection terms. It can be approximated by 1 for symmetrical wafer structures.

The total radiative recombination coefficient $B_{\mathrm{rad}}(T)$ is determined from integration by

$$
\begin{aligned}
B_{\mathrm{rad}}(T) & =\int B_{\mathrm{rad}, \mathrm{s}}(T, \hbar \omega) \mathrm{d}(\hbar \omega) \\
& =\frac{c^{\prime}}{n p} \int \mathrm{d} j_{\mathrm{PL}}(T, \hbar \omega)=\frac{c^{\prime}}{n p} J_{\mathrm{PL}}(T)
\end{aligned}
$$

here, the term $c^{\prime}=\frac{4 n_{r}^{2}(T, \hbar \omega)}{C_{\text {ref }} d}$ has been extracted from the integral because $C_{\text {ref }}$ and the related $n_{r}(T, \hbar \omega)$ do not vary much in the spectral energy range of the PL emission [8]. In addition $c^{\prime}$ is taken constant as the $T$-dependence is small even down to $20 \mathrm{~K}$ [8].

Between the measured signal $S$, delivered by the detector, and the emitted $J_{\mathrm{PL}}(T)$ another calibration factor, $c^{\prime \prime}$ here, may be introduced that relates the measured current or voltage to the photon flux. A $T$ and energy independent factor $c^{\prime \prime}$ represents this contribution via $S=c^{\prime \prime} J_{\mathrm{PL}}$.

And thus $B_{\text {rad }}(T)$ can be deduced from $S$ and from the carrier densities by

$$
B_{\mathrm{rad}}(T)=\frac{c S(T)}{n p}
$$

with $c=c^{\prime} / c^{\prime \prime}$. The value of $c$ can be chosen to calibrate the relative $B_{\text {rad }}$-values with literature data in order to determine absolute $B_{\text {rad }}$-values.

For the specific case of an n-type wafer

$$
B_{\mathrm{rad}}(T) \approx \frac{c S(T)}{\left[\Delta n(T)+n_{0}(T)\right] \Delta p(T)}
$$

with the excess carrier densities $\Delta n$ and $\Delta p$ and the thermal densities $n_{0} \gg p_{0}$ and $\Delta p \gg p_{0}$.

For determining the excess carrier densities in Eq. (9), MPL is measured by illuminating the c-Si wafer with sinusoidally modulated light which is related to a photogeneration rate $G$ according $G=G_{D}+G_{A} \exp (i \omega t)$, where $G_{\mathrm{D}}$ represents the bias-illumination induced DC generation rate, $G_{\mathrm{A}}$ is the amplitude of the AC modulation and $\omega$ the modulation angular frequency. The average $G$-value is determined from the ratio of $F_{\mathrm{abs}}$, the portion of photon flux absorbed by the sample, and $d$.

In steady state, with $G=G_{\mathrm{D}}$, a recombination lifetime $\tau$ can be defined according to $G_{D}=\Delta p_{D} / \tau$, with the stationary excess density $\Delta p_{D}$. Based on the Ansatz $\Delta p=\Delta p_{D}+\Delta p_{A} \exp (i \omega t)$, in which $\Delta p_{A}$ is complex, a phase shift $\phi$ of the PL signal with respect to the modulated generation is derived with $[9,10]$

$$
\phi=-\arctan \left(\omega \tau_{\mathrm{MPL}}\right) .
$$

with an effective carrier lifetime $\tau_{\mathrm{MPL}}$. In the derivation a $G$ independent $\tau$ is assumed such that $\tau_{\text {MPL }}$ equals $\tau$. This is quite reasonable for our samples as will be discussed later together with the presentation of the experimental data.

At last, the excess carrier densities $\Delta n(T)$ and $\Delta p(T)$ can be deduced from the MPL lifetime with

$$
\Delta p(T)=\Delta n(T)=G_{D} \tau_{\mathrm{MPL}}(T)=\frac{F_{\mathrm{abs}}}{d} \tau_{\mathrm{MPL}}(T) .
$$

For Eq. (9) we need to determine the equilibrium electron density $n_{0}(T)$, by taking the temperature 
dependence of the ionization of the donors with density $N_{d}$ into consideration. We calculate $n_{0}(T)$ numerically from the charge neutrality equation and the Fermi-level dependent occupation of the conduction band. Thus, $n_{0}(T)$ equals the ionized donor density $N_{d}^{+}$according to $[11]$

$$
n_{0} \approx N_{d}^{+}=\frac{N_{d}}{1+2 \mathrm{e}^{\left(E_{f}-E_{d}\right) / k T}},
$$

and is also determined by

$$
n_{0}=N_{c} \exp \left(-\frac{E_{c}-E_{f}}{k T}\right),
$$

where $E_{f}$ is the $T$-dependent Fermi level, $E_{d}$ the donor activation energy, $N_{c}$ the $T$-dependent effective density of states in the conduction band and $E_{c}$ the energy of conduction band edge. We take $E_{c}-E_{d}=45 \mathrm{meV}$ as reported for phosphorus in silicon [12] and $N_{d}$ according to the conductance values of the phosphorus doped c-Si wafers under investigation.

With $S(T), \Delta n(T), \Delta p(T)$ and $n_{0}(T)$ the relative radiative recombination coefficient $B_{\text {rad }}$ can be obtained with Eq. (9) as a function of temperature. It is noted that a

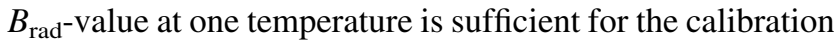
to absolute values.

The experimental setup is equipped with a closedcycle $\mathrm{He}$ cryostat which allows cooling down to $20 \mathrm{~K}$. A 785-nm laser diode illuminates the sample through the cryostat window using an optical fiber. The laser power is measured with an Ophir Nova II photometer at the position of the sample. The emitted luminescence is collected in reflection mode by a parabolic mirror and focused with an optical lens onto the monochromator entrance. The optics part is a $4 f$ system with a magnification of one. A highpass optical filter is placed in front of the monochromator to filter the reflected or scattered excitation light. An InGaAs photodiode measures the dispersed spectral photon flux at each given wavelength. The photocurrent is amplified and converted into voltage to be measured by a Stanford Research SR830 lock-in amplifier or a Keithley 195 digital multimeter. For the MPL, a sinusoidal output signal of the lock-in amplifier modulates the laser-diode current. Test measurements with fixed $G_{\mathrm{D}}$ and a variation

Table 1 Description of the two c-Si wafer samples.

\begin{tabular}{lll}
\hline & sample A & sample B \\
\hline $\mathrm{c}-\mathrm{Si}$ & $\mathrm{Cz}$ & $\mathrm{Fz}$ \\
$d(\mu \mathrm{m})$ & 130 & 280 \\
surface & textured & polished \\
$\rho(\Omega \mathrm{cm})$ & 9 & 2.6 \\
passivation & $50 \mathrm{~nm} \mathrm{AlO}_{x}$ (both sides), & $50 \mathrm{~nm}$ intrinsic \\
& $\mathrm{SiC}$ on rear side & a-Si:H (both sides) \\
$R @ 785 \mathrm{~nm}$ & 0.012 & 0.38 \\
\hline
\end{tabular}

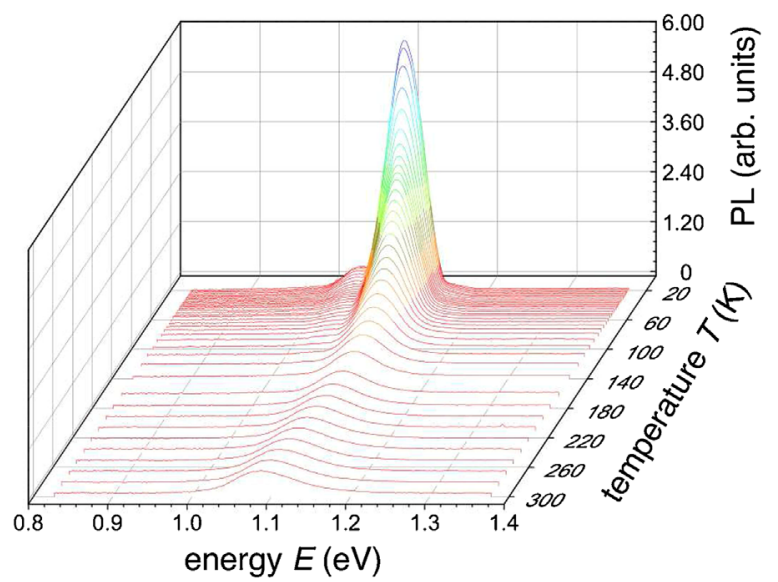

Figure $1 T$-dependent PL spectra of sample A between 300 and $20 \mathrm{~K}$.

of $G_{A}$ showed that the phase was independent of the $G_{A} / G_{D^{-}}$ratio.

We investigate the PL spectra and MPL lifetimes of two different c-Si wafers, a sample from a $\mathrm{Cz}-\mathrm{Si}$ wafer labelled sample $\mathrm{A}$ and from an $\mathrm{Fz}-\mathrm{Si}$ wafer, labelled sample B. Table 1 summarizes their properties with different thicknesses $d$, textures, doping in terms of resistivity $\rho$ and passivation schemes as well as the reflectance $R$ at the excitation wavelength. Low laserpower excitation values in the range of 0.5 and $1 \mathrm{~mW} \mathrm{~cm}^{-2}$ were used.

Exemplarily, the PL spectra of sample A are illustrated in Fig. 1 as a function of temperature between 300 and $20 \mathrm{~K}$. The corresponding integrated PL intensities $S(T)$ as a function of temperature are shown in Fig. 2.

Figure 3(a) presents the MPL lifetimes of samples A and B. Data for sample B were taken from four consecutive runs of the MPL and sPL-measurements, when measuring from 315 to $20 \mathrm{~K}$, followed by a 20 to $315 \mathrm{~K}$ sequence, and by a repetition of this cycle. The standard deviation was determined and is plotted in Fig. 3(a). The carrier densities $\Delta p(T)=\Delta n(T)$, determined from the MPL-lifetimes are depicted together with the calculated $n_{0}(T)$ in Fig. 3(b).

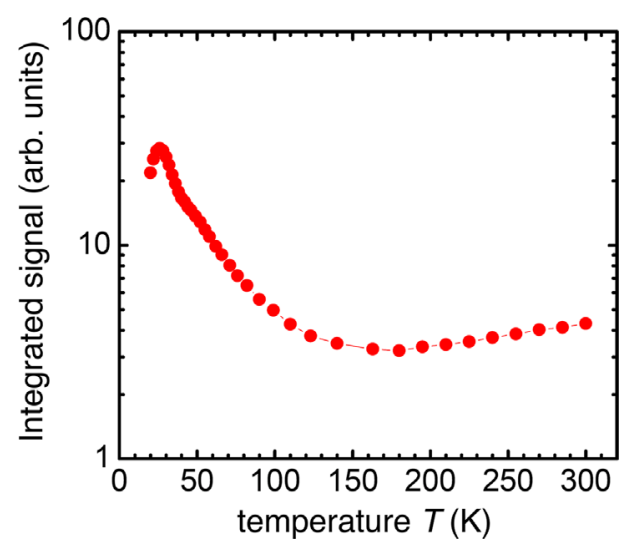

Figure 2 Integrated PL signal $S(T)$ as a function of $T$. 

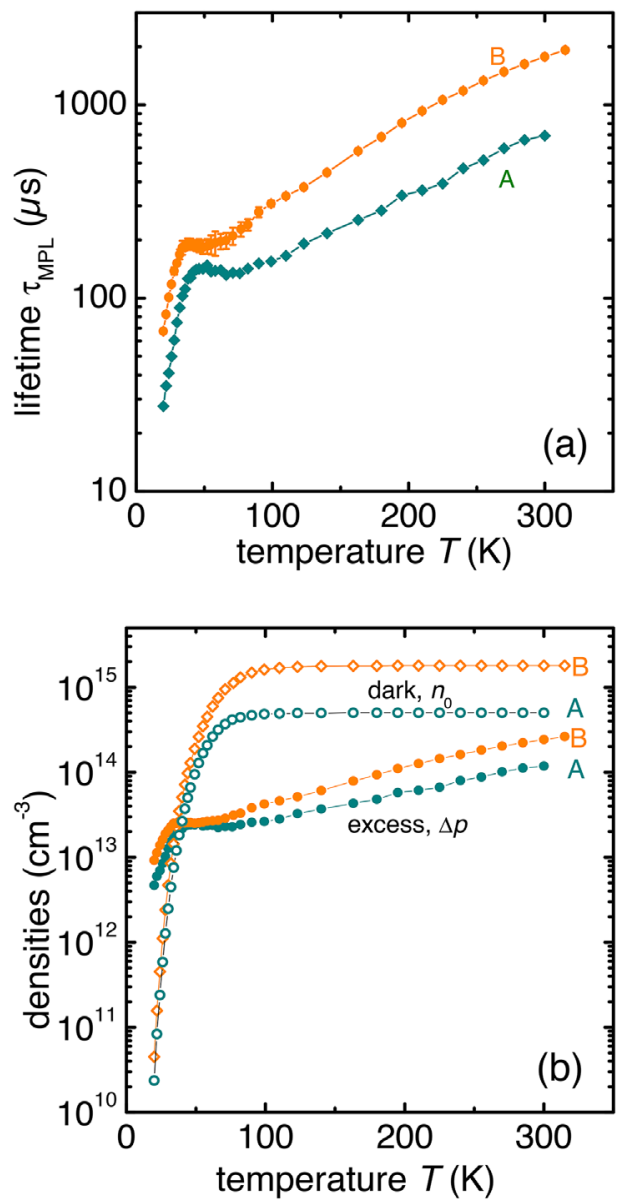

Figure 3 In (a), MPL lifetimes of samples A and B versus $T$ are shown. For sample $\mathrm{B}$ the mean values and error bars are determined from four consecutive measurements from two down/ up-cycles in $T$. For most of the temperatures the error bars are smaller than the symbol size. In (b), the densities $\Delta p(=\Delta n)$, determined from the MPL lifetimes and $n_{0}$ of samples A and B versus $T$ are shown.

The hole density $p_{0}(T)$ at equilibrium is negligible in these n-type wafers. The determined excess carrier density is well below the doping density, respectively.

In a next step we determine the relative values of the radiative recombination coefficient $B_{\text {rad }}(T)$ according to Eq. (9) from our measured integrated PL data and from the determined carrier densities. One value for $c$ is adjusted by matching the relative $B_{\mathrm{rad}}(T)$ with literature data [1] thus calibrating $B_{\text {rad }}(T)$.

The resulting $B_{\mathrm{rad}}(T)$ for the two samples A and B are summarized in Fig. 4 in which the available $T$ range for $B_{\text {rad }}$ is extended below $77 \mathrm{~K}$ towards $20 \mathrm{~K}$. Values for $B_{\text {rad }}$ have not been available so far in this $T$ range. For $T \geq 77 \mathrm{~K}$ our data are consistent with the literature with a maximum deviation of about $20 \%$ at around $170 \mathrm{~K}$.

The data for sample B show the mean value from the four runs of the MPL and sPL-measurements. The relative difference between samples $A$ and $B$ is

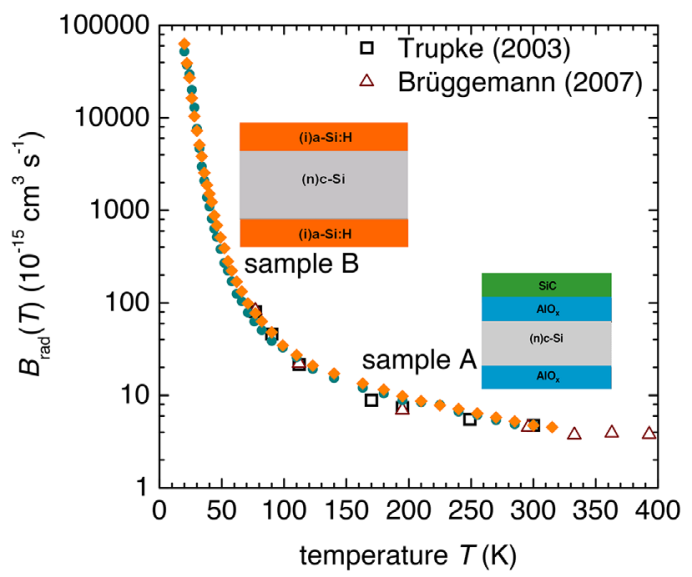

Figure 4 Radiative recombination coefficient versus temperature for samples A (diamonds) and B (circles). The structures are sketched in the figure. For sample B the mean values from the four $T$-dependent measurements are shown. Results from [3] are similar to the literature data shown (squares and triangles) from $[1,2]$.

temperature dependent and around 10\% for most temperatures. It amounts to around $40 \%$ in a narrow range around $50 \mathrm{~K}$.

At this stage the possible difference between the lifetime from MPL as a differential lifetime and the steadystate lifetime, for example [14], is briefly discussed. Such a difference may occur if the excess carrier density does not vary linearly with generation rate. For sample A such dependence according to $\tau \propto \Delta p^{\beta}$ has shown a low value of $\beta$ of around 0.14 to 0.16 at $300 \mathrm{~K}$ from quasi-steady state photoconductance. For c-Si wafers passivated with intrinsic a-Si:H this $\beta$-value is known to be much less and closer to zero. According to [14] one can expect deviations between differential and effective lifetime to be less than $20 \%$ for sample A and much less for sample B. However, should the ratio of differential lifetime and steady-state lifetime not be unity but temperature independent, Eq. (8) will care that this ratio is absorbed in the factor $c$, which would not introduce an additional error since $B_{\text {rad }}$ was calibrated at $300 \mathrm{~K}$. Should the ratio be temperature dependent, which is unknown, there would be an error that is introduced in the determination of $B_{\mathrm{rad}}$. Such an error may well account for the $20 \%$ observed variation between literature values and those from the present method. It is noted that at low temperatures the large variation and strong increase in $B_{\text {rad }}$ is well above any error from a possibly temperature-dependent ratio of MPL lifetime and steady-state lifetime.

Despite the different properties of the two wafer samples with respect to deposition, thickness and doping, good agreement is achieved for the two sets of $B_{\mathrm{rad}}$-data, despite the accounted differences of maximal $40 \%$ around $50 \mathrm{~K}$ but with better agreement $<20 \%$ at temperatures higher and lower than $50 \mathrm{~K}$. The surface passivation layers of $\mathrm{AlO}_{x}$ and intrinsic a-Si:H lead to different band bending in the crystalline silicon 
space-charge regions of the wafers. Fixed negative charge in the $\mathrm{AlO}_{x}$ [13] leads to electron depletion and a different band bending as in the space charge regions of the a-Si:H passivated wafer and different lifetimes in the two samples. Nevertheless, the PL-related results with respect to $B_{\text {rad }}$ are not strongly influenced so that it may be concluded that the volumes of the c-Si wafers are probed for the $B_{\text {rad }}$-determination.

In conclusion, we used a combination of MPL and SPL measurements to determine the radiative recombination coefficient $B_{\text {rad }}$ in crystalline silicon. MPL allows us to determine the lifetime from the phase shift with reference to the modulated excitation. The $T$-dependence of $B_{\text {rad }}$ can then be extracted from the $T$-dependence of the measured lifetime and SPL signal. Compared to previous determinations of $B_{\text {rad }}$ our approach does not require the precise knowledge of the $T$-dependence of the absorption coefficient or the intrinsic carrier density. One issue in our approach could be the potential discrepancy between the differential lifetime measured by MPL and the steadystate lifetime determining the sPL. However assuming that these two lifetimes are equal we achieved acceptable agreement between our $B_{\text {rad }}$ values and those found in the literature in the temperature range where such data were available $(T \geq 77 \mathrm{~K})$, with less than $20 \%$ difference in the whole $T$-range. Moreover, we could extend the measurement range for $B_{\text {rad }}$-values from 77 to $20 \mathrm{~K}$ and identified an increase by about three orders of magnitude with decreasing temperature. This observation could stimulate further experimental and theoretical work on the $T$-dependence of $B_{\text {rad }}$ at low $T$ range.

Acknowledgements This work was partly supported by the project HERCULES funded from the European Unions
Seventh Programme for Research Technological Development and Demonstration under Grant agreement no. 608498. M. X. thanks the China Scholarship Council for the $\mathrm{PhD}$ grant. The authors are grateful to I. Martín (UPC, Barcelona) and to I. Sobkowicz (LPICM, Palaiseau) for providing passivated silicon wafers.

\section{References}

[1] T. Trupke, M. A. Green, P. Würfel, P. P. Altermatt, A. Wang, J. Zhao, and R. Corkish, J. Appl. Phys. 94(8), 4930 (2003).

[2] R. Brüggemann, J. Behrends, S. Meier, and S. Tardon, J. Optoelectron. Adv. Mater. 9(1), 77-83 (2007).

[3] H. T. Nguyen, S. C. Baker-Finch, and D. Macdonald, Appl. Phys. Lett. 104(11), 112105 (2014).

[4] M. Ruff, M. Fick, R. Lindner, U. Rössler, and R. Helbig, J. Appl. Phys. 74, 267 (1993).

[5] P. Würfel, J. Phys. C: Solid State Phys. 15(18), 3967 (1982).

[6] P. Würfel, S. Finkbeiner, and E. Daub, Appl. Phys. A 60(1), 67-70 (1995).

[7] T. Trupke, E. Daub, and P. Würfel, Sol. Energy Mater. Sol. Cells 53(1-2), 103-114 (1998).

[8] B. J. Frey, D. B. Leviton, and T. J. Madison, Proc. SPIE 6273, 62732J (2006).

[9] D. K. Schroder, Semiconductor Material and Device Characterization (Wiley, New York, 1990).

[10] R. Brüggemann and S. Reynolds, J. Non-Cryst. Solids 352(9-20), 1888-1891 (2006).

[11] R. F. Pierret, Advanced Semiconductor Fundamentals (Prentice Hall, Upper Saddle River, NJ, 2003), Chap. 4.4.

[12] S. M. Sze and K. K. Ng, Physics of Semiconductor Devices (John Wiley \& Sons, Hoboken, NJ, 2006).

[13] F. Werner, B. Veith, D. Zielke, L. Kühnemund, C. Tegenkamp, M. Seibt, R. Brendel, and J. Schmidt, J. Appl. Phys. 109(11), 113701 (2011).

[14] J. A. Giesecke, S. W. Glunz, and W. Warta, J. Appl. Phys. 113(7), 073706 (2013). 